\title{
Study of a mixed problem for a nonlinear elasticity system by topological degree
}

\author{
Zoubai Fayrouz and Merouani Boubakeur
}

\begin{abstract}
In this paper, we consider a mixed problem for a nonlinear elasticity system with laws of general behavior. The coefficients of elasticity depends on $x$ meanwhile the density of the volumetric forces depends on the displacement. The main aim of this paper is to apply the Schauder's fixed point theorem and the techniques of topological degree to prove a theorem of the existence and the uniqueness of the solution of the corresponding variational problem.
\end{abstract}

Mathematics Subject Classification (2010): 35J45, 35J55, 35A05, 35A07, 35A15.

Keywords: Boundary conditions, nonlinear elasticity, mixed problem, Schauder's fixed point theorem, topological degree, existence and uniqueness.

\section{Introduction}

This work consists in solving the mixed problem for the nonlinear elasticity system, by means of two methods, namely, the theorem of Schauder and the techniques of the topological degree [7].

First, we introduce the following notations needed in this paper. Let $\Omega$ be a connected open bounded domain of $\mathbb{R}^{N},(N=3)$ with Lipschitz boundary $\Gamma$. Let $\Gamma_{0}$ a part of $\Gamma$ of strictly positive superficial measure, and let $\Gamma_{1}$ be the complement of $\Gamma_{0}$ in $\Gamma$. For a given field of displacement $u$, we associate a linearized displacement tensor $\varepsilon(u)$ defined by

$$
\varepsilon(\nabla u(x))=\frac{1}{2}\left(\nabla^{T} u+\nabla u\right)
$$

whose components are

$$
\varepsilon_{i j}(u(x))=\frac{1}{2}\left(\frac{\partial u_{i}}{\partial x_{j}}+\frac{\partial u_{j}}{\partial x_{i}}\right), 1 \leq i, j \leq 3 .
$$


The corresponding constraints tensor $\sigma(u)$ is given by

$$
\sigma_{i j}(u(x))=\sum_{k, h=1}^{3} a_{i j k h}(x) \varepsilon_{k h}(u(x)), 1 \leq i, j \leq 3 .
$$

Equation (1.2) describes a linear relation between the stress tensor $\left\{\sigma_{i j}\right\}$ and the deformation tensor $\left\{\varepsilon_{i j}\right\}$. The elasticity coefficients $a_{i j k h}$ satisfy the following properties:

1. Properties of symmetry:

$$
a_{i j k h}=a_{j i k h}=a_{i j h k}, \forall 1 \leq i, j, k, h \leq 3 ;
$$

2. Property of ellipticity:

$$
\exists \alpha>0, \forall\left\{\xi_{i j}\right\} \in \mathbb{R}^{N^{2}}, \sum_{k, h=1}^{3} a_{i j k h} \xi_{i j} \xi_{k h} \geq \alpha \sum_{i, j=1}^{3} \xi_{i j}^{2} .
$$

\section{Position of problem}

We consider a fundamental example of a nonlinear elliptic problem derived from the Mechanics of Solids, namely, the nonlinear elasticity system. Let $f$ be such that $f(x, u(x))=\left(f_{1}(x, u(x)), f_{2}(x, u(x)), f_{3}(x, u(x))\right)$ of $\left(L^{2}(\Omega)\right)^{3}$ and $g=\left(g_{1}, g_{2}, g_{3}\right)$ of $\left(L^{2}\left(\Gamma_{1}\right)\right)^{3}$, the problem is to find a function $u=\left(u_{1}, u_{2}, u_{3}\right)$ solution of the nonlinear elliptic problem:

$$
\begin{gathered}
-\sum_{j=1}^{3} \frac{\partial}{\partial x_{j}} \sigma_{i j}(u)=f_{i}(x, u) \quad \text { in } \quad \Omega ; \quad \forall 1 \leq i \leq 3 ; \\
u_{i}=0 \quad \text { on } \quad \Gamma_{0} ; \quad \forall 1 \leq i \leq 3 ; \\
\sum_{j=1}^{3} \sigma_{i j}(u) \eta_{j}=g_{i} \quad \text { on } \quad \Gamma_{1} ; \quad \forall 1 \leq i \leq 3 .
\end{gathered}
$$

Equations (2.1), (2.2) and (2.3) describe the small displacements $u$ from the natural state of a non-homogeneous elastic solid subjected to a volume density of forces $f$ in $\Omega$, and to a superficial density of forces $g$ on $\Gamma_{1}$, the displacements $u$ being fixed by zero on $\Gamma_{0}$, i.e., $\left.\gamma u\right|_{\Gamma_{0}}=0$.

Several authors studied the system of elasticity with laws of particular behavior and using various techniques for example in [1], Ciarlet used the implicit function theorem to show the existence and uniqueness of a solution. Dautry-Lions [2], studied the linear problem in a regular boundary domain. Later on, Merouani in [6], [4], [5], studied the Lamé (elasticity) system in a polygonal boundary domain.

The bibliography quoted here does not claim to be exhaustive and the deficiency must be attributed to the author's ignorance and not to the author's ill will.

The tensor of the constraints considered here is linear and grouped, as special cases, some models used in Ciarlet [1], Lions [3] and Dautry-Lions [2]. Let us cite by the way the examples: 
1. The problem of pure displacement for a homogeneous or heterogeneous material of St Vennan-Kirchhoff where:

- The applied volumetric forces $f$ are dead (does not depend on $u$ ),

- The tensor of stress is in the form (material of St Vennan-Kirchhoff ) where

$$
\left\{\begin{array}{c}
\sigma_{i j}(u(x))=\lambda\left(\operatorname{tr} E_{i j}(\nabla u(x))\right)+2 \mu E_{i j}(\nabla u(x)) \\
1 \leq i, j \leq 3, \lambda>0, \mu>0
\end{array}\right.
$$

2. The coefficients of elasticity have the form:

$$
a_{i j p q}=\lambda \delta_{i j} \delta_{p q}+\mu\left(\delta_{i p} \delta_{j q}+\delta_{i q} \delta_{j p}\right), 1 \leq i, j, p, q \leq 3
$$

with, $\lambda$ and $\mu$ depend on $x$ or not.

3. The applied volumetric forces $f$ have the form

$$
f(\xi)=|\xi|^{p-1} \xi, 1<p<\infty .
$$

The material is not homogeneous, we assume that the functions $a_{i j k h}$ belong to $L^{\infty}(\Omega), 1 \leq i, j, k, h \leq N$ and the elliptic property is uniform, there exist a constant $\alpha>0$, independent of $x$, such that (1.4) is verified almost everywhere on $\Omega$.

\section{Weak formulation}

We suppose that the solution $u$ of $(2.1)-(2.3)$ exists and belongs to $\left(H^{2}(\Omega)\right)^{3}$. Multiply the equation $(2.1)$ by $v \in V$, and integrate on $\Omega$, we obtain:

$$
-\int_{\Omega} \sum_{j=1}^{3} \frac{\partial}{\partial x_{j}} \sigma_{i j}(u) v_{i}(x) d x=\int_{\Omega} f_{i}(x, u(x)) v_{i}(x) d x
$$

where

$$
V=\left\{v \in\left(H^{1}(\Omega)\right)^{3} ; v=0 \text { in } \Gamma_{0}\right\},
$$

is a closed vector subspace of $\left(H^{1}(\Omega)\right)^{3}$, equipped with the norm $\|\cdot\|_{V}=\|\cdot\|_{\left(H^{1}(\Omega)\right)^{3}}$. By Green's formula, we have

$$
\int_{\Omega} \sum_{i, j=1}^{3} \sigma_{i j}(u) \frac{\partial v_{i}}{\partial x_{j}} d x-\int_{\Gamma} \sum_{i, j=1}^{3} \sigma_{i j}(u) \eta_{j} v_{i} d \Gamma=\sum_{i=1}^{3} \int_{\Omega} f_{i}(x, u(x)) v_{i}(x) d x,
$$

which implies

$$
\begin{aligned}
\int_{\Omega} \sum_{i, j=1}^{3} \sum_{k, h=1}^{3} a_{i j k h}(x) \varepsilon_{k h}(u) \varepsilon_{i j}(v) d x & =\int_{\Omega} f(x, u(x)) v(x) d x \\
& +\int_{\Gamma_{1}} g(x) v(x) d \Gamma, \forall v \in V .
\end{aligned}
$$




\section{Existence theorem}

\subsection{Existence with Schauder's theorem}

Let us first recall the notion of Caratheodory function.

Definition 4.1. (Function of Caratheodory): Let $N, p, q \in \mathbb{N}^{*}$ and $\Omega$ an open set of $\mathbb{R}^{N}$. Let $a$ be an application of $\Omega \times \mathbb{R}^{p}$ to $\mathbb{R}^{q}$. We say that $a$ is a Caratheodory function if $a(; s)$ is a Borel function for all $s$ of $\mathbb{R}^{p}$ and $a(x ;)$ is continuous for almost all $x$ of $\Omega$.

In this section, we need the following assumptions:

$$
\left\{\begin{array}{l}
\Omega \text { is a connected open bounded domain of } \mathbb{R}^{N}, \\
\text { with Lipschitz boundary } \Gamma ; \\
\exists \alpha>0 \text { and } \beta>0 \text { such as } \alpha \leq a_{i j k h}(s) \leq \beta \text { a.e. for all } s \in \mathbb{R} ; \\
f \in\left(L^{\infty}(\Omega \times \mathbb{R})\right)^{3} ; \\
\varepsilon_{i j} \text { is a continuous function, } \forall 1 \leqslant i, j \leqslant 3 .
\end{array}\right.
$$

Under the assumptions (4.1), we try to show the existence of $u$, the solution of the following nonlinear problem:

$$
\left\{\begin{array}{c}
u \in V \\
\int_{\Omega} \sum_{i, j=1}^{3} \sum_{k, h=1}^{3} a_{i j k h}(x) \varepsilon_{k h}(u(x)) \varepsilon_{i j}(v(x)) d x \\
=\int_{\Omega} f(x, u(x)) v(x) d x+\int_{\Gamma_{1}} g(x) v(x) d \Gamma, \forall v \in V
\end{array}\right.
$$

Theorem 4.2. Under the assumptions (4.1), there exist a solution $u$ of the problem $(P)$.

Proof. For $\bar{u} \in\left(L^{2}(\Omega)\right)^{3}$, we have the existence and the uniqueness of the solution $u$ of the following problem:

$$
\left\{\begin{array}{c}
u \in V \\
\int_{\Omega} \sum_{i, j=1}^{3} \sum_{k, h=1}^{3} a_{i j k h}(x) \varepsilon_{k h}(u(x)) \varepsilon_{i j}(v(x)) d x \\
=\int_{\Omega} f(x, \bar{u}(x)) v(x) d x+\int_{\Gamma_{1}} g(x) v(x) d \Gamma, \forall v \in V .
\end{array}\right.
$$

More precisely, to show the existence and uniqueness of $u$, the solution of $(P 1)$, we apply the Lax-Milgram Lemma [1]. Let $T(\bar{u})=u$, where $T$ is an application of $E$ in $E$ with

$$
E=\left(L^{2}(\Omega)\right)^{3}
$$

A fixed point of $T$ is a solution of the problem $(P)$. To prove the existence of such a fixed point, we apply the Schauder's fixed point theorem. First, we will show that 
the image of $T$ lies in a bounded of $V$. Using $\alpha$ cited in hypothesis (4.1), we have

$$
\left\{\begin{array}{l}
\alpha \int_{\Omega} \sum_{i, j=1}^{3} \sum_{k, h=1}^{3} \varepsilon_{k h}(u(x)) \varepsilon_{i j}(v(x)) d x \\
\leq \int_{\Omega} \sum_{i, j=1}^{3} \sum_{k, h=1}^{3} a_{i j k h}(x) \varepsilon_{k h}(u(x)) \varepsilon_{i j}(v(x)) d x \\
=\int_{\Omega} f(x, \bar{u}(x)) v(x) d x+\int_{\Gamma_{1}} g(x) v(x) d \Gamma .
\end{array}\right.
$$

Taking $v=u$ in (4.2), and using Korn's inequality [8], we obtain

$$
\alpha C\|u\|_{\left(H^{1}(\Omega)\right)^{3}}^{2} \leq \int_{\Omega} f(x, \bar{u}(x)) u(x) d x+\int_{\Gamma_{1}} g(x) u(x) d \Gamma .
$$

By Cauchy-Schwartz inequality, the bound $L^{\infty}$ of $f$ and the trace theorem, we get,

$$
\begin{aligned}
& \alpha C\|u\|_{\left(H^{1}(\Omega)\right)^{3}}^{2} \leq C_{1}\|u\|_{\left(L^{2}(\Omega)\right)^{3}}+\|g\|_{\left(L^{2}\left(\Gamma_{1}\right)\right)^{3}}\|u\|_{\left(L^{2}\left(\Gamma_{1}\right)\right)^{3}} \\
& \alpha C\|u\|_{\left(H^{1}(\Omega)\right)^{3}}^{2} \leq C_{1}\|u\|_{\left(H^{1}(\Omega)\right)^{3}}+C_{2} C_{3}\|u\|_{\left(H^{1}(\Omega)\right)^{3}},
\end{aligned}
$$

which implies

$$
\|u\|_{V}=\|u\|_{\left(H^{1}(\Omega)\right)^{3}} \leq \frac{C_{1}+C_{2} C_{3}}{\alpha C}=R,
$$

thus

$$
\|u\|_{\left(L^{2}(\Omega)\right)^{3}} \leq R
$$

so

$$
u \in B_{R}=\left\{u \in\left(L^{2}(\Omega)\right)^{3} /\|u\|_{\left(L^{2}(\Omega)\right)^{3}} \leq R\right\} .
$$

And as a result, the image of $T$ is in a bounded of $V \subset\left(H^{1}(\Omega)\right)^{3}$. By Rellich's theorem the image of $T$ is in a compact of $\left(L^{2}(\Omega)\right)^{3}$. Taking $R$ large enough, therefore, the application $T$ sends $B_{R}$ in $B_{R}$ and $\left\{T(\bar{u}), \bar{u} \in B_{R}\right\}$ is relatively compact in $\left(L^{2}(\Omega)\right)^{3}$. To apply Schauder's fixed point theorem, it remains to show the continuity of $T$. Let $\left(\bar{u}_{n}\right)_{n \in \mathbb{N}}$ a sequence of $\left(L^{2}(\Omega)\right)^{3}$ such as $\bar{u}_{n} \rightarrow \bar{u}$ in $\left(L^{2}(\Omega)\right)^{3}$, when $n \rightarrow+\infty$. Letting $u_{n}=T\left(\bar{u}_{n}\right)$. After extracting a subsequence, we can assume that $\bar{u}_{n} \rightarrow \bar{u}$ a.e., and that there exist $w \in V$ such that $u_{n} \rightarrow w$ weakly in $V$ and so $u_{n} \rightarrow w$ strongly in $\left.\left(L^{2}(\Omega)\right)^{3}\right)$. Now, we will show that $w$ is the solution of the problem $(P 1)$. Indeed, let $v \in V$, we have

$$
\begin{aligned}
\int_{\Omega} \sum_{i, j=1}^{3} \sum_{k, h=1}^{3} a_{i j k h}(x) \varepsilon_{k h}\left(u_{n}(x)\right) \varepsilon_{i j}(v(x)) d x & =\int_{\Omega} f\left(x, \bar{u}_{n}\right) v(x) d x \\
& +\int_{\Gamma_{1}} g(x) v(x) d \Gamma, \forall v \in V
\end{aligned}
$$


Passing to the limit when $n \rightarrow+\infty$ (using Dominated Convergence Theorem), we will have

$$
\begin{aligned}
\int_{\Omega} \sum_{i, j=1}^{3} \sum_{k, h=1}^{3} a_{i j k h}(x) \varepsilon_{k h}(w) \varepsilon_{i j}(v(x)) d x & =\int_{\Omega} f(x, \bar{u}(x)) v(x) d x \\
& +\int_{\Gamma_{1}} g(x) v(x) d \Gamma, \forall v \in V .
\end{aligned}
$$

This proves that $w=T(\bar{u})=u$. We have thus proved, after extraction of a subsequence, that $T\left(\bar{u}_{n}\right) \rightarrow T(\bar{u})$ in $\left(L^{2}(\Omega)\right)^{3}$. By the absurd one can show that this convergence remains true without extraction of subsequence. Thus, we have proved the continuity of $T$. Therefore, we can apply the Schauder's fixed point theorem and to conclude that there is a fixed point of $T$, which ends the proof.

\subsection{Existence by topological degree}

We take again the same previous problem:

$$
\left\{\begin{array}{c}
u \in V \\
\int_{\Omega} \sum_{i, j=1}^{3} \sum_{k, h=1}^{3} a_{i j k h}(x) \varepsilon_{k h}(u(x)) \varepsilon_{i j}(v(x)) d x \\
=\int_{\Omega} f(x, u(x)) v(x) d x+\int_{\Gamma_{1}} g(x) v(x) d \Gamma, \forall v \in V
\end{array}\right.
$$

which is the weak formulation of the problem (2.1)-(2.3).

The following assumptions are made.

$$
\left\{\begin{array}{l}
(i) \Omega \text { is a connected open bounded domain of } \mathbb{R}^{N}, \\
\text { with Lipchitez boundary } \Gamma, \\
(i i) \varepsilon_{i j} \text { is a continuous function } \forall 1 \leqslant i, j \leqslant 3, \\
\left(\text { iii } \exists \alpha \text { and } \beta>0 \text {; such that } \alpha \leq a_{i j k h}(x) \leq \beta \text { a.e. on } \Omega,\right. \\
(i v) f \text { is a Carathéodory function, and } \exists C_{2} \geq 0 \text { and } d \in\left(L^{2}(\Omega)\right)^{3} \\
|f(x, s)| \leq d(x)+C_{2}|s|, \\
(v) \lim _{s \rightarrow \infty} \frac{f(x, s)}{s}=0 .
\end{array}\right.
$$

Theorem 4.3. Under the assumptions (4.3), there exist a solution of the problem $(P)$. In addition, if $f$ does not depend to $u$, then the solution is unique.

Proof. The method of the topological degree requires a priory estimates, i.e., the estimates on $u$, without knowing its existence. We therefore suppose that $u$ is a solution of $(P)$. The great advantage of considering $(P)$ rather than $(P 1)$ is to have only $u$, not $u$ and $\bar{u}$, and this greatly simplifies the estimates. 
We rewrite the problem $(P)$ under the following form:

$$
\left\{\begin{array}{c}
u \in V \\
\int \sum_{\Omega} \sum_{i, j=1}^{3} \sum_{k, h=1}^{3} a_{i j k h}(x) \varepsilon_{k h}(u(x)) \varepsilon_{i j}(v(x)) d x=\langle F(u), v\rangle_{V^{\prime}, V}
\end{array}\right.
$$

where $F(u)$ is, for $u \in\left(L^{2}(\Omega)\right)^{3}$, the element of $V^{\prime}$ defined by

$$
\langle F(u), v\rangle_{V^{\prime}, V}=\int_{\Omega} f(x, u(x)) v(x) d x+\int_{\Gamma_{1}} g(x) v(x) d \Gamma .
$$

According to the hypothesis $(i v)$, the Cauchy-Schwartz inequality and the trace theorem, we have

$$
\begin{aligned}
|\langle F(u), v\rangle| & \leq \int_{\Omega}|f(x, u(x))| \cdot|v(x)| d x+\int_{\Gamma_{1}}|g(x)| \cdot|v(x)| d \Gamma \\
& \leq \int_{\Omega}\left|d(x)+C_{2}\right| u|| \cdot|v| d x+\int_{\Gamma_{1}}|g(x)| \cdot|v(x)| d \Gamma \\
& \leq \int_{\Omega}|d(x)| \cdot|v| d x+C_{2} \int_{\Omega}|u||v| d x+\int_{\Gamma_{1}}|g(x)||v(x)| d \Gamma \\
& \leq\|d\|_{L^{2}(\Omega)^{3}}\|v\|_{L^{2}(\Omega)^{3}}+C_{2}\|u\|_{L^{2}(\Omega)^{3}}\|v\|_{L^{2}(\Omega)^{3}}+\|g\|_{L^{2}\left(\Gamma_{1}\right)^{3}}\|v\|_{L^{2}\left(\Gamma_{1}\right)^{3}} \\
& \leq\|d\|_{\left(L^{2}(\Omega)\right)^{3}}\|v\|_{\left(H^{1}(\Omega)\right)^{3}}+C_{2}\|u\|_{\left(L^{2}(\Omega)\right)^{3}}\|v\|_{\left(H^{1}(\Omega)\right)^{3}}+C_{0} C \|_{\left(H^{1}(\Omega)\right)^{3}} \\
& \leq\left[\|d\|_{\left(L^{2}(\Omega)\right)^{3}}+C_{2} R+C C_{0}\right]\|v\|_{V} \cdot
\end{aligned}
$$

Then

$$
\|F(u)\|_{V^{\prime}} \leq\|d\|_{\left(L^{2}(\Omega)\right)^{3}}+C_{2} R+C C_{0} .
$$

We deduce that $F(u)$ is an element of $V^{\prime}$, for any $u \in\left(L^{2}(\Omega)\right)^{3}$.

We will show that

$$
\begin{gathered}
F:\left(L^{2}(\Omega)\right)^{3} \rightarrow V^{\prime} \\
u \longmapsto F(u)
\end{gathered}
$$

is continuous. For this, we need the Lebesgue Dominated Convergence Theorem.

Let $u, \widetilde{u} \in\left(L^{2}(\Omega)\right)^{3}$; we have

$$
\begin{aligned}
\langle F(u), v\rangle & =\int_{\Omega} f(x, u(x)) v(x) d x+\int_{\Gamma_{1}} g(x) v(x) d \Gamma \\
\langle F(\widetilde{u}), v\rangle & =\int_{\Omega} f(x, \widetilde{u}(x)) v(x) d x+\int_{\Gamma_{1}} g(x) v(x) d \Gamma,
\end{aligned}
$$


SO

$$
\begin{aligned}
\|F(u)-F(\widetilde{u})\|_{V^{\prime}} & =\sup _{\substack{v \in V \\
\|v\|=1}}\langle F(u)-F(\widetilde{u}), v\rangle_{V^{\prime}, V} \\
& =\sup _{\substack{u \in V \\
\|v\|=1}}\left[\int_{\Omega}(f(u)-f(\widetilde{u})) \cdot v d x\right] \\
& \leq \sup _{\substack{\|\in V\\
\| v \|=1}}\left[\|f(u)-f(\widetilde{u})\|_{\left(L^{2}(\Omega)\right)^{3}} \cdot\|v\|_{\left(L^{2}(\Omega)\right)^{3}}\right] \\
& \leq \sup _{\substack{v \in V \\
\|v\|=1}}\left[\|f(u)-f(\widetilde{u})\|_{\left(L^{2}(\Omega)\right)^{3}}\|v\|_{V}\right] \\
& \leq\|f(u)-f(\widetilde{u})\|_{\left(L^{2}(\Omega)\right)^{3}} .
\end{aligned}
$$

So, if $\left(u_{n}\right)_{n \in \mathbb{N}}$ is a sequence of $\left(L^{2}(\Omega)\right)^{3}$ such that $u_{n} \rightarrow \widetilde{u}$ in $\left(L^{2}(\Omega)\right)^{3}$, we have

$$
\left\|F\left(u_{n}\right)-F(\widetilde{u})\right\|_{V^{\prime}} \leq\left\|f\left(u_{n}\right)-f(\widetilde{u})\right\|_{\left(L^{2}(\Omega)\right)^{3}} .
$$

So, $\exists\left(u_{n}\right)$ subsequence such that

$$
u_{n} \rightarrow \widetilde{u}(x) \text { almost everywhere in } \Omega
$$

and $\exists H \in\left(L^{2}(\Omega)\right)^{3}$ such that

$$
\left|u_{n}\right| \leq H \text { almost everywhere in } \Omega .
$$

Then, we notice that $f\left(u_{n}\right) \rightarrow f(\widetilde{u})$ because $f$ is continuous a.e. in $\Omega$. According to the hypothesis $(i v),\left|f\left(u_{n}\right)\right| \leq d(x)+C_{2}\left|u_{n}\right|$, and as $\left|u_{n}\right| \leq H$ we find

$$
\left|f\left(u_{n}\right)\right| \leq d(x)+C_{2} \cdot H \text { almost everywhere in } \Omega .
$$

So, by the Lebesgue Dominated Convergence Theorem, we obtain

$$
\left\|f\left(u_{n}\right)-f(\widetilde{u})\right\|_{\left(L^{2}(\Omega)\right)^{3}} \rightarrow 0 \text { when } n \rightarrow \infty
$$

and consequently,

$$
\left\|F\left(u_{n}\right)-F(\widetilde{u})\right\|_{V^{\prime}} \rightarrow 0 \text { when } n \rightarrow \infty
$$

hence the continuity of $F$. For $S \in V^{\prime}$, the linear problem

$$
\left\{\begin{array}{c}
w \in V \\
\int \sum_{\Omega} \sum_{i, j=1}^{3} \sum_{k, h=1}^{3} a_{i j k h}(x) \varepsilon_{k h}(w(x)) \varepsilon_{i j}(v(x)) d x=\langle S, v\rangle_{V^{\prime}, V},
\end{array}\right.
$$

admits a unique solution $w \in V$ (see [1]). We denote by $B_{u}$ the operator which to $S$ in $V^{\prime}$ associates $w$ solution of (4.5). The operator $B_{u}$ is linear continuous from $V^{\prime}$ into $V$ and $V$ is injected compactly into $\left(L^{2}(\Omega)\right)^{3}$ (because the boundary $\Gamma$ is lipschitzian). We deduce that the operator $B_{u}$ is compact from $V^{\prime}$ in $\left(L^{2}(\Omega)\right)^{3}$. The problem $(P)$ 
is equivalent to solving the fixed point problem $u=B_{u}(F(u))$. We will show, using the topological degree techniques, that the following problem admits a solution

$$
\left\{\begin{array}{c}
u \in\left(L^{2}(\Omega)\right)^{3}, \\
u=B_{u}(F(u)) .
\end{array}\right.
$$

For $t \in[0,1]$, we put the application $h$ such that:

$$
\begin{gathered}
h:[0,1] \times\left(L^{2}(\Omega)\right)^{3} \rightarrow\left(L^{2}(\Omega)\right)^{3} \\
(t, u) \longmapsto h(t, u)=B_{u}(t F(u))
\end{gathered}
$$

For $R>0$, we put $B_{R}=\left\{u \in\left(L^{2}(\Omega)\right)^{3}\right.$ such that $\left.\|u\|_{\left(L^{2}(\Omega)\right)^{3}}<R\right\}$. We will show

$(1)-\exists R>0 ;\left\{\begin{array}{l}u-h(t, u)=0 \\ t \in[0,1], u \in\left(L^{2}(\Omega)\right)^{3}\end{array}\right\} \Longrightarrow\|u\|_{\left(L^{2}(\Omega)\right)^{3}}<R$;

$(2)-h$ is continuous from $[0,1] \times \bar{B}_{R}$ into $\bar{B}_{R}$;

$(3)-\left\{h(t, u), t \in[0,1], u \in \bar{B}_{R}\right\}$ is relatively compact in $\left(L^{2}(\Omega)\right)^{3}$.

If we suppose that we have proved the statements $(1),(2)$ and $(3)$, we have no solution to the equation $u-h(t, u)=0$ on the boundary of the ball $B_{R}$, and we can thus define the degree $d\left(I d-h(t,),. B_{R}, 0\right)$. This degree does not depend of $t$, so we have

$$
\begin{aligned}
d\left(I_{d}-h(t, .), B_{R}, 0\right) & =d\left(I d-h(0, .), B_{R}, 0\right) \\
& =d\left(I d, B_{R}, 0\right)=1 .
\end{aligned}
$$

We deduce the existence of $u \in B_{R}$ such that $u-h(1, u)=0$, that is to say

$$
u=B_{u}(F(u)) \text {. }
$$

Thus $u$ is solution of $(P)$. Now, it remains to show the statements (1), (2) and (3). Let us begin with the proof of (3) (for every $R>0$ ). We suppose $\|u\|_{\left(L^{2}(\Omega)\right)^{3}} \leq R$. We have

$$
F(u) \in V^{\prime}, \text { and }\langle F(u), v\rangle_{V^{\prime}, V}=\int_{\Omega} f(x, u(x)) v(x) d x+\int_{\Gamma_{1}} g(x) v(x) d \Gamma .
$$

We have

$$
\|F(u)\|_{V^{\prime}} \leq\|d\|_{\left(L^{2}(\Omega)\right)^{3}}+C_{2} R+C C_{0} .
$$

So

$$
t\|F(u)\|_{V^{\prime}} \leq\|d\|_{\left(L^{2}(\Omega)\right)^{3}}+C_{2} R+C C_{0}=\widetilde{R}, \forall t \in[0,1]
$$

We put $h(t, u)=B_{u}(t F(u))=w$ and show that there exists $\bar{R}$ depending only of $R$, $C_{0}, C, C_{2}$, and $\alpha$ such that

$$
\|h(t, u)\|_{V} \leq \bar{R} \Longleftrightarrow\|w\|_{V} \leq \bar{R}
$$

By definition, $w$ is solution of

$$
\left\{\begin{array}{l}
w \in V \\
\int_{\Omega} \sum_{i, j=1}^{3} \sum_{k, h=1}^{3} a_{i j k h}(x) \varepsilon_{k h}(w(x)) \varepsilon_{i j}(v(x)) d x \\
=\langle t F(u), v\rangle_{V^{\prime}, V}, \forall v \in V
\end{array}\right.
$$


Taking $v=w$ in (4.6), by Korn's inequality, we obtain,

$$
\begin{gathered}
\alpha k\|w\|_{\left(H^{1}(\Omega)\right)^{3}}^{2} \leq\|t F(u)\|_{V^{\prime}}\|w\|_{V} \\
\Longleftrightarrow \alpha k\|w\|_{\left(H^{1}(\Omega)\right)^{3}}^{2} \leq\|t F(u)\|_{V^{\prime}}\|w\|_{V} \leq \widetilde{R}\|w\|_{V}
\end{gathered}
$$

which implies:

$$
\|h(t, u)\|_{V}=\|w\|_{V} \leq \bar{R}
$$

with

$$
\bar{R}=\frac{\widetilde{R}}{\alpha k}=\frac{\|d\|_{\left(L^{2}(\Omega)\right)^{3}}+C_{2} R+C C_{0}}{\alpha k} .
$$

From Rellich's Theorem, we deduce that the set $\left\{h(t, u), t \in[0,1], u \in \bar{B}_{R}\right\}$ is relatively compact in $\left(L^{2}(\Omega)\right)^{3}$, which shows (3). Let us show now the point (2). Let $\left(t_{n}\right)_{n \in \mathbb{N}} \subset[0,1]$ such that $t_{n} \rightarrow t$ when $n \rightarrow+\infty$ and $\left(u_{n}\right)_{n \in \mathbb{N}} \subset\left(L^{2}(\Omega)\right)^{3}$ with $u_{n} \rightarrow u$ in $\left(L^{2}(\Omega)\right)^{3}$. We want to show that $h\left(t_{n}, u_{n}\right) \rightarrow h(t, u)$ in $\left(L^{2}(\Omega)\right)^{3}$. Let $w_{n}=h\left(t_{n}, u_{n}\right)$ and $w=h(t, u)$. To show that $w_{n} \rightarrow w$ in $\left(L^{2}(\Omega)\right)^{3}$. We take the limit on the following problem,

$$
\left\{\begin{array}{c}
\int_{\Omega_{n} \in V} \sum_{i, j=1}^{3} \sum_{k, h=1}^{3} a_{i j k h}(x) \varepsilon_{k h}\left(w_{n}\right) \varepsilon_{i j}(v) d x \\
=t_{n} \int_{\Omega} f\left(u_{n}\right) v(x) d x+t_{n} \int_{\Gamma_{1}} g(x) v(x) d \Gamma
\end{array}\right.
$$

We already know that $\left(w_{n}\right)_{n \in \mathbb{N}}$ is bounded in $V$, because the sequence $\left(u_{n}\right)_{n \in \mathbb{N}}$ is bounded in $\left(L^{2}(\Omega)\right)^{3}$ (this is what was shown in the previous step: if $\left\|u_{n}\right\|_{\left(L^{2}(\Omega)\right)^{3}} \leq R$ then $\left.\left\|w_{n}\right\|_{V} \leq \bar{R}\right)$. The sequence $\left(w_{n}\right)_{n \in \mathbb{N}}$ is bounded in $V$, and to a subsequence, we have

$$
\begin{aligned}
& w_{n} \rightarrow \bar{w} \text { in } V \text { weakly and } w_{n} \rightarrow \bar{w} \text { in }\left(L^{2}(\Omega)\right)^{3}, \\
& u_{n} \rightarrow u \text { a.e. and } \exists H \in\left(L^{2}(\Omega)\right)^{3} ;\left|u_{n}\right| \leq H \text { a.e.. }
\end{aligned}
$$

Since $w_{n} \rightarrow \bar{w}$ in $\left(L^{2}(\Omega)\right)^{3}$, then there exist a subsequence denoted again $w_{n}$ such that

$$
w_{n} \rightarrow \bar{w} \text { a.e. and } \exists K \in\left(L^{2}(\Omega)\right)^{3} ;\left|w_{n}\right| \leq K \text { a.e. }
$$

Let $v \in V$ and as $\varepsilon_{k h}$ is continuous then $\varepsilon_{k h}\left(w_{n}\right) \rightarrow \varepsilon_{k h}(\bar{w})$ a.e. and so

$$
\sum_{i, j=1}^{3} \sum_{k, h=1}^{3} a_{i j k h}(x) \varepsilon_{k h}\left(w_{n}\right) \varepsilon_{i j}(v) \rightarrow \sum_{i, j=1}^{3} \sum_{k, h=1}^{3} a_{i j k h}(x) \varepsilon_{k h}(\bar{w}) \varepsilon_{i j}(v) \text { a.e. }
$$

we have also

$$
\left|\sum_{i, j=1}^{3} \sum_{k, h=1}^{3} a_{i j k h}(x) \varepsilon_{k h}\left(w_{n}\right) \varepsilon_{i j}(v)\right| \leq \beta \sum_{k, h=1}^{3}\left|\varepsilon_{k h}\left(w_{n}\right)\right| \sum_{i, j=1}^{3}\left|\varepsilon_{i j}(v)\right| \in L^{1}(\Omega),
$$


by Dominated Convergence Theorem, we have

$$
\int_{\Omega} \sum_{i, j=1}^{3} \sum_{k, h=1}^{3} a_{i j k h}(x) \varepsilon_{k h}\left(w_{n}\right) \varepsilon_{i j}(v) d x \rightarrow \int_{\Omega} \sum_{i, j=1}^{3} \sum_{k, h=1}^{3} a_{i j k h}(x) \varepsilon_{k h}(\bar{w}) \varepsilon_{i j}(v) d x, n \rightarrow+\infty \text {. }
$$

As $f\left(u_{n}\right) \rightarrow f(u)$ a.e. and $\left|f\left(u_{n}\right)\right| \leq|d|+C_{2}|H|$.

By the Lebesgue Dominated Convergence Theorem we have $f\left(u_{n}\right) \rightarrow f(u)$ in $\left(L^{2}(\Omega)\right)^{3}$ and consequently

$$
\int_{\Omega} f\left(u_{n}\right) v d x \rightarrow \int_{\Omega} f(u) v d x
$$

when $n \rightarrow+\infty$. Passing to the limit in (4.7), we obtain,

$$
\int_{\Omega} \sum_{i, j=1}^{3} \sum_{k, h=1}^{3} a_{i j k h}(x) \varepsilon_{k h}(\bar{w}) \varepsilon_{i j}(v) d x=t \int_{\Omega} f(u) v d x+t \int_{\Gamma_{1}} g(x) v(x) d \Gamma,
$$

and so $\bar{w}=h(t, u)=w$. By the absurd argument, we show that $w_{n} \rightarrow w$ in $V$ weakly and $w_{n} \rightarrow w$ in $\left(L^{2}(\Omega)\right)^{3}$, where $w_{n}=h\left(t_{n}, u_{n}\right)$ and $w=h(t, u)$; the application $h$ is continuous and consequently (2) holds. It remains now to demonstrate (1). We want to show that:

$$
\exists R>0 ;\left\{\begin{array}{c}
u-h(t, u)=0 \\
t \in[0,1], u \in\left(L^{2}(\Omega)\right)^{3}
\end{array}\right\} \Longrightarrow\|u\|_{\left(L^{2}(\Omega)\right)^{3}}<R .
$$

Let $t \in[0,1]$ and $u=h(t, u)=t B_{u}(F(u))$, that is to say

$$
\left\{\begin{array}{l}
\int_{\Omega} \sum_{i, j=1}^{3} \sum_{k, h=1}^{3} a_{i j k h}(x) \varepsilon_{k h}(u) \varepsilon_{i j}(v) d x \\
t=\int_{\Omega} f(u) v d x+t \int_{\Gamma_{1}} g(x) v(x) d \Gamma, \forall v \in V \\
u \in V
\end{array}\right.
$$

We choose $v=u$ in (4.8). By the hypotheses (4.3), and Korn's inequality, we have

$$
\alpha k\|u\|_{\left(H^{1}(\Omega)\right)^{3}}^{2} \leq \int_{\Omega}|f(u) u| d x+\int_{\Gamma_{1}}|g(x)||u(x)| d \Gamma .
$$

We are going to deduce from this inequality the existence of $R>0$ such that

$$
\|u\|_{\left(L^{2}(\Omega)\right)^{3}}<R .
$$

Here, we use the hypothesis $(v)$, i.e.,

$$
\lim _{s \rightarrow \pm \infty} \frac{f(x, s)}{s}=0 .
$$

We argue by the absurd. Let us suppose that a such $R$ does not exist. Then there exist a sequence $\left(u_{n}\right)_{n \in \mathbb{N}^{*}}$ of elements of $V$ such that

$$
\left\|u_{n}\right\|_{\left(L^{2}(\Omega)\right)^{3}} \geq n \text { and } \alpha k\left\|u_{n}\right\|_{\left(H^{1}(\Omega)\right)^{3}}^{2} \leq \int_{\Omega}\left|f\left(u_{n}\right) u_{n}\right| d x+\int_{\Gamma_{1}}|g(x)|\left|u_{n}(x)\right| d \Gamma \text {. }
$$


Let us show that this is impossible. Letting $v_{n}=\frac{u_{n}}{\left\|u_{n}\right\|_{V}}$. We have $\left\|v_{n}\right\|_{V}=1$ and

$$
\alpha k\left\|v_{n}\right\|_{\left(H^{1}(\Omega)\right)^{3}}^{2} \leq \int_{\Omega}\left|\frac{f\left(u_{n}\right)}{\left\|u_{n}\right\|_{\left(H^{1}(\Omega)\right)^{3}}} v_{n}\right| d x+\int_{\Gamma_{1}}\left|\frac{g(x)}{\left\|u_{n}\right\|_{\left(H^{1}(\Omega)\right)^{3}}} v_{n}\right| d \Gamma .
$$

Or, according to hypothesis (iv) i.e., $|f(x, s)| \leq d(x)+C_{2}|s|$ and the trace theorem we have

$$
\begin{aligned}
\alpha k\left\|v_{n}\right\|_{\left(H^{1}(\Omega)\right)^{3}}^{2} & \leq \int_{\Omega} \frac{|d|+C_{2}\left|u_{n}\right|}{\left\|u_{n}\right\|_{\left(H^{1}(\Omega)\right)^{3}}}\left|v_{n}\right| d x+\frac{\|g(x)\|_{\left(L^{2}\left(\Gamma_{1}\right)\right)^{3}}\left\|v_{n}\right\|_{\left(L^{2}\left(\Gamma_{1}\right)\right)^{3}}}{\left\|u_{n}\right\|_{\left(H^{1}(\Omega)\right)^{3}}} \\
& \leq \int_{\Omega} \frac{|d|\left|v_{n}\right|}{\left\|u_{n}\right\|_{\left(H^{1}(\Omega)\right)^{3}}} d x+C_{2} \int_{\Omega}\left|v_{n}\right|^{2} d x+\frac{\|g(x)\|_{\left(L^{2}\left(\Gamma_{1}\right)\right)^{3}} C\left\|v_{n}\right\|_{\left(H^{1}(\Omega)\right)^{3}}}{\left\|u_{n}\right\|_{\left(H^{1}(\Omega)\right)^{3}}} \\
& \leq \frac{\|d\|_{\left(L^{2}(\Omega)\right)^{3}}\left\|v_{n}\right\|_{\left(L^{2}(\Omega)\right)^{3}}}{\left\|u_{n}\right\|_{\left(H^{1}(\Omega)\right)^{3}}}+C_{2}\left\|v_{n}\right\|_{\left(L^{2}(\Omega)\right)^{3}}^{2}+\frac{C_{0} C}{\left\|u_{n}\right\|_{\left(H^{1}(\Omega)\right)^{3}}} \\
& \leq \frac{\|d\|_{\left(L^{2}(\Omega)\right)^{3}}\left\|v_{n}\right\|_{\left(L^{2}(\Omega)\right)^{3}}}{\left\|u_{n}\right\|_{\left(L^{2}(\Omega)\right)^{3}}}+C_{2}\left\|v_{n}\right\|_{\left(H^{1}(\Omega)\right)^{3}}^{2}+\frac{C_{0} C}{\left\|u_{n}\right\|_{\left(L^{2}(\Omega)\right)^{3}}} \\
& \leq\|d\|_{\left(L^{2}(\Omega)\right)^{3}}\left\|v_{n}\right\|_{\left(L^{2}(\Omega)\right)^{3}}+C_{2}+C_{0} C \\
& \leq\|d\|_{\left(L^{2}(\Omega)\right)^{3}}+C_{2}+C_{0} C,
\end{aligned}
$$

which implies

$$
\left\|v_{n}\right\|_{V}^{2} \leq \frac{\|d\|_{\left(L^{2}(\Omega)\right)^{3}}+C_{2}+C_{0} C}{\alpha k},
$$

so, $\left(v_{n}\right)_{n \in \mathbb{N}^{*}}$ is bounded in $V$, and hence there exist a subsequence, $v_{n} \rightarrow v$ in $\left(L^{2}(\Omega)\right)^{3}$. We also have

$$
\begin{aligned}
v_{n} & \rightarrow v \text { a.e. on } \Omega \\
\left|v_{n}\right| & \leq H \text { with } H \in\left(L^{2}(\Omega)\right)^{3}
\end{aligned}
$$

As $\left\|v_{n}\right\|_{V}=1$, we have

$$
\alpha k \leq \int_{\Omega}\left|\frac{f\left(u_{n}\right)}{\left\|u_{n}\right\|_{\left(H^{1}(\Omega)\right)^{3}}} v_{n}\right| d x+\int_{\Gamma_{1}}\left|\frac{g(x)}{\left\|u_{n}\right\|_{\left(H^{1}(\Omega)\right)^{3}}} v_{n}\right| d \Gamma .
$$

Letting

$$
X_{n}=\int_{\Omega}\left|\frac{f\left(u_{n}\right)}{\left\|u_{n}\right\|_{\left(H^{1}(\Omega)\right)^{3}}} v_{n}\right| d x+\int_{\Gamma_{1}}\left|\frac{g(x)}{\left\|u_{n}\right\|_{\left(H^{1}(\Omega)\right)^{3}}} v_{n}\right| d \Gamma \text {. }
$$

Now, we show that $X_{n} \rightarrow 0$ when $n \rightarrow+\infty$, which is impossible since $X_{n}$ is reduced by the constant $\alpha k$ which is strictly positive. 
- Let us show that $\frac{\left|f\left(u_{n}\right)\right|\left|v_{n}\right|}{\left\|u_{n}\right\|_{\left(H^{1}(\Omega)\right)^{3}}} \rightarrow 0$ a.e. with domination, we shall have then by the Dominated Convergence Theorem that $\int_{\Omega}\left|\frac{f\left(u_{n}\right)}{\left\|u_{n}\right\|_{\left(H^{1}(\Omega)\right)^{3}}} v_{n}\right| d x \rightarrow 0$ when $n \rightarrow+\infty$.

We show first of all the domination. We have

$$
\begin{aligned}
\frac{\left|f\left(u_{n}\right)\right|}{\left\|u_{n}\right\|_{\left(H^{1}(\Omega)\right)^{3}}} & \leq \frac{|d|+C_{2}\left|u_{n}\right|}{\left\|u_{n}\right\|_{\left(H^{1}(\Omega)^{3}\right.}} \leq \frac{|d|}{\left\|u_{n}\right\|_{\left(H^{1}(\Omega)^{3}\right.}}+C_{2}\left|v_{n}\right| \\
& \leq \frac{|d|}{\left\|u_{n}\right\|_{\left(L^{2}(\Omega)\right)^{3}}}+C_{2}\left|v_{n}\right| \\
& \leq|d|+C_{2} H
\end{aligned}
$$

so

$$
\left|\frac{f\left(u_{n}\right)}{\left\|u_{n}\right\|_{\left(H^{1}(\Omega)\right)^{3}}} v_{n}\right| \leq\left(|d|+C_{2} H\right) H \in\left(L^{1}(\Omega)\right)^{3} .
$$

Now, we show that the convergence is almost everywhere. We have $v_{n} \rightarrow v$ a.e. so $\exists A ; \operatorname{mes}\left(A^{c}\right)=0$ and $v_{n}(x) \rightarrow v(x) \forall x \in A$.

Case 1. If $v(x)>0 ; v_{n}(x) \rightarrow v(x)$, but

$$
\lim _{n \rightarrow+\infty}\left\|u_{n}\right\|_{\left(L^{2}(\Omega)\right)^{3}}=+\infty
$$

SO

$$
\begin{gathered}
u_{n}(x)=v_{n}(x)\left\|u_{n}\right\|_{V} \rightarrow+\infty . \\
\frac{f\left(u_{n}(x)\right)}{\left\|u_{n}\right\|_{\left(H^{1}(\Omega)\right)^{3}}} v_{n}(x)=\frac{f\left(u_{n}(x)\right) u_{n}(x)}{u_{n}(x)\left\|u_{n}\right\|_{\left(H^{1}(\Omega)\right)^{3}}} v_{n}(x)=\frac{f\left(u_{n}(x)\right)}{u_{n}(x)}\left(v_{n}(x)\right)^{2} \rightarrow 0, n \rightarrow+\infty .
\end{gathered}
$$

We used here $\lim _{s \rightarrow+\infty} f(s) / s=0$.

Case 2. If $v(x)<0$; we have the same

$$
\lim _{n \rightarrow+\infty} \frac{f\left(u_{n}(x)\right)}{\left\|u_{n}\right\|_{\left(H^{1}(\Omega)\right)^{3}}} v_{n}(x)=0
$$

because

$$
\lim _{s \rightarrow-\infty} f(s) / s=0 .
$$

Case 3. If $v(x)=0$;

$$
\begin{aligned}
\left|\frac{f\left(u_{n}(x)\right)}{\left\|u_{n}\right\|_{\left(H^{1}(\Omega)\right)^{3}}} v_{n}(x)\right| & \leq \frac{|d(x)|+C_{2}\left|u_{n}(x)\right|}{\left\|u_{n}\right\|_{\left(H^{1}(\Omega)\right)^{3}}}\left|v_{n}(x)\right| \\
& \leq\left(|d(x)|+C_{2}\left|v_{n}(x)\right|\right)\left|v_{n}(x)\right| \\
& \rightarrow 0 \text { because } v(x)=0 .
\end{aligned}
$$

In summary, we have

$$
\frac{f\left(u_{n}\right)}{\left\|u_{n}\right\|_{\left(H^{1}(\Omega)\right)^{3}}} v_{n} \rightarrow 0
$$


a.e. on $\Omega$. It has been shown that

$$
\lim _{n \rightarrow+\infty} \int_{\Omega}\left|\frac{f\left(u_{n}\right)}{\left\|u_{n}\right\|_{\left(H^{1}(\Omega)\right)^{3}}} v_{n}\right| d x=0 .
$$

- Let us now show that the term $\int_{\Gamma_{1}}\left|\frac{g(x)}{\left\|u_{n}\right\|_{\left(H^{1}(\Omega)\right)^{3}}} v_{n}\right| \rightarrow 0$ as $n \rightarrow+\infty$.

We have

$$
\begin{aligned}
0 \leq \int_{\Gamma_{1}} \frac{|g(x)|\left|v_{n}\right|}{\left\|u_{n}\right\|_{\left(H^{1}(\Omega)\right)^{3}}} d \Gamma & \leq \frac{\|g(x)\|_{\left(L^{2}\left(\Gamma_{1}\right)\right)^{3}}\left\|v_{n}\right\|_{\left(L^{2}\left(\Gamma_{1}\right)\right)^{3}}}{\left\|u_{n}\right\|_{\left(H^{1}(\Omega)\right)^{3}}} \\
& \leq \frac{C_{0} C\left\|v_{n}\right\|_{\left(H^{1}(\Omega)\right)^{3}}}{\left\|u_{n}\right\|_{\left(H^{1}(\Omega)\right)^{3}}} \\
& \leq \frac{C_{0} C}{\left\|u_{n}\right\|_{\left(L^{2}(\Omega)\right)^{3}}} \rightarrow 0 \text { when } n \rightarrow+\infty
\end{aligned}
$$

Because we have $\left\|u_{n}\right\|_{\left(L^{2}(\Omega)\right)^{3}} \rightarrow+\infty$ when $n \rightarrow+\infty$. It has been shown that

$$
\lim _{n \rightarrow+\infty} \int_{\Gamma_{1}} \frac{|g(x)|\left|v_{n}\right|}{\left\|u_{n}\right\|_{\left(H^{1}(\Omega)\right)^{3}}} d \Gamma=0 .
$$

So $\lim _{n \rightarrow+\infty} X_{n}=0$, which is a contradiction with $X_{n} \geq \alpha k$ for all $n \in \mathbb{N}^{*}$. Thus, we have showed that there is $R>0$ such as: $(u=h(t, u)) \Longrightarrow\|u\|_{\left(L^{2}(\Omega)\right)^{3}}<R$. This proves (1). Then the existence of solution to $(P)$ is proved.

Uniqueness. We suppose that $f$ does not depend to $u$. Let $u_{1}$ and $u_{2}$ be two solutions of this problem:

$$
\begin{aligned}
& \int_{\Omega} \sum_{i, j=1}^{3} \sum_{k, h=1}^{3} a_{i j k h}(x) \varepsilon_{k h}\left(u_{i}\right) \varepsilon_{i j}(v) d x \\
= & \int_{\Omega} f(x) v(x) d x+\int_{\Gamma_{1}} g(x) v(x) d \Gamma, \quad i=1,2 ; \forall v \in V .
\end{aligned}
$$

Subtracting term to term and substituting $v$ by $u_{1}-u_{2}$, we obtain,

$$
\int_{\Omega} \sum_{i, j=1}^{3} \sum_{k, h=1}^{3} a_{i j k h}(x)\left(\varepsilon_{k h}\left(u_{1}-u_{2}\right)\right) \varepsilon_{i j}\left(u_{1}-u_{2}\right) d x=0,
$$

by Korn's inequality, and the hypothesis (iii), we have

$$
\alpha\left\|u_{1}-u_{2}\right\|_{\left(H^{1}(\Omega)\right)^{3}}^{2} \leq 0,
$$

so, $u_{1}=u_{2}$. This completes the proof of Theorem 4.3. 


\section{Conclusion}

In this work, we have studied the existence and the uniqueness of solutions of the mixed problem for a nonlinear elasticity system in a regular and bounded domain by using Schauder's fixed point theorem and the technique of topological degree. Next future work, we will concentrate on the same problem but with $\varepsilon$ is nonlinear, and we will also prove a theorem of existence and uniqueness of solutions in Sobolev spaces with variable exponents.

\section{References}

[1] Ciarlet, P.G., Mathematical elasticity, Vol. I: Three-Dimensional Elasticity, NorthHolland, Amsterdam, 1988.

[2] Dautray, R., Lions, J.L., Analyse Mathématique et Calcul Numérique pour les Sciences et les Techniques, Vol. 1, Masson, 1984.

[3] Lions, J.L., Quelques Méthode de Résolution des Problèmes aux Limites non Linéaires, Dunod, Paris, 1969.

[4] Merouani, B., Solutions singulières du système de l'élasticité dans un polygone pour différentes conditions aux limites, Maghreb Math. Rev., 5(1996), no. 1 \& 2, 95-112.

[5] Merouani, B., Quelques problèmes aux limites pour le système de Lamé dans un secteur plan, C.R.A.S., T. 304, Série I, no. 13, 1987.

[6] Merouani, B., Boufenouche, R., Trigonometric series adapted for the study of Dirichlet boundary-value problems of Lamé systems, Electron. J. Differential Equations, 2015(2015), no. 181, 1-6.

[7] Rabinowitz, P.H., Théorie du degré topologique et application à des problèmes aux limites non linéaires (Lecture note by H. Berestycki), Report 75010, Laboratoire d'analyse Numériqe, Université Pierre et Marie Curie, Paris, 1989.

[8] Raviart, P.A., Tomas, J.M., Introduction à l'Analyse Numériques des Équations aux Dérivées Partielles, Masson, Paris, 1983.

Zoubai Fayrouz

Setif 1 University, Department of Mathematics, Applied Mathemathics Laboratory (LaMA), 19000, Algeria

e-mail: fayrouz.zoubai@univ-setif.dz

Merouani Boubakeur

Setif 1 University, Department of Mathematics, Applied Mathemathics Laboratory (LaMA), 19000, Algeria

e-mail: mermathsb@hotmail.fr 